\begin{tabular}{l|l|l|l}
$\begin{array}{c}\text { Case Reports in } \\
\text { Demillatology }\end{array}$ & $\begin{array}{l}\text { Case Rep Dermatol 2011;3:55-59 } \\
\text { DOI: } 10.1159 / 000326055\end{array}$ & $\begin{array}{l}\text { Published online: } \\
\text { March 4, 2011 }\end{array}$ & $\begin{array}{l}\text { I 2011 S. Karger AG, Basel } \\
\text { ISSN 1662-6567 } \\
\text { www.karger.com/cde }\end{array}$ \\
\hline
\end{tabular}

\title{
Tinea incognito Caused by Microsporum gypseum in a Patient with Advanced HIV Infection: A Case Report
}

\author{
Ennio Polillia Paolo Fazii ${ }^{a}$ Tamara Ursini ${ }^{b}$ \\ Fabrizio Fantinic Francesco Di Masi ${ }^{\mathrm{b}}$ \\ Monica Tontodonati ${ }^{b}$ Federica Sozio ${ }^{b}$ Giustino Parruti ${ }^{b}$ \\ Units of a Clinical Pathology and ${ }^{b}$ Infectious Disease, Pescara General Hospital, \\ Pescara, and 'Department of Dermatology, University of Modena and Reggio \\ Emilia, Modena, Italy
}

\section{Key Words}

HIV infection - Tinea incognito $\cdot$ Microsporum gypseum

\begin{abstract}
The prevalence and the clinical relevance of dermatophytoses in HIV-infected patients are poorly documented, particularly for those caused by tinea incognito. Here, we report a case of widespread facial tinea incognito occurring in an Italian patient with advanced HIV infection, showing both skin and brain lesions. Second-line treatment with liposomal amphotericin B and cotrimoxazole, administered after a microbiological characterization of the skin scrapings, led to complete clearance of all lesions.
\end{abstract}

\section{Introduction}

Dermatophytoses are localized fungal infections of the skin, interdigital space, groin and hair; they are very common in the general population and do not usually cause serious clinical diseases [1]. The prevalence of dermatophytoses in HIV-infected patients is poorly documented and may be underestimated in those living in developed countries, due to the early start of antiretroviral therapy in recent years and to the frequent use of azoles, both for prophylaxis and therapy of other fungal diseases [2].

Tinea incognito is a peculiar dermatophytosis with altered, polymorphic manifestations, lacking most of the typical morphological features that characterize tinea corporis, including round or oval presentation, sharp edges, vesicles, and scaling. It is commonly due to topical immunosuppressive agents, like steroids. Tinea incognito is frequently misdiagnosed as psoriasis, contact dermatitis and other types of eczema, 
rosacea, and lupus erythematosus. Cultures from tinea incognito usually yield the same dermatophytes isolated from typical dermatophytoses, the most frequently reported in Europe being Trichophyton rubrum, Trichophyton mentagrophytes, Microsporum canis, Epidermophyton floccosum, and Microsporum gypseum [3, 4].

In the present study, we report a case of diffuse and persistent tinea incognito caused by M. gypseum in a patient with advanced HIV disease experiencing clinical progression and an isolated brain lesion.

\section{Case Report}

A 37-year-old male drug addict was diagnosed with HIV and HCV infection in 1996. Antiretroviral therapy was started in 1997 , when his CD4 T-cell counts were $236 / \mathrm{mm}^{3}$ and his viral load was 23,104 copies/ml. His adherence to antiretrovirals was suboptimal until 2005. He carried on a dual therapy with zidovudine and didanosine at standard doses until 1998, when he was switched to his first highly active antiretroviral therapy (HAART) regimen including stavudine, lamivudine, nevirapine and nelfinavir. He remained on this HAART regimen with poor adherence until clinical progression late in 2005 . His HIV viral load never exceeded 30,000 copies/ml; CD4 T-cell counts ranged from 30 to $120 / \mathrm{mm}^{3}$, with liver function tests always in the normal range. During this period, he worked as a floor-cleaning assistant.

In absence of any signs of clinical progression, the patient came to our institution in May 2005, due to dermatological lesions confined to his face and neck. Lesions consisted of annular erythematous scaly plaques with inflammatory advancing borders located on the upper lip, nose and side of the neck (fig. 1). Dermatophytosis was clinically diagnosed, and oral itraconazole, $200 \mathrm{mg}$ b.i.d., was prescribed until September 2005, when his condition had completely cleared. Thus, we decided to stop the antifungal therapy. The patient missed a few programmed checks. In January 2006, he came again to our institution because of a relapse of his facial disease. Lesions consisted of ill-defined, confluent, poorly inflammatory erythematous scaly patches, diffusely involving the face and neck (fig. 2a, b). Itraconazole at the same dosage ( $400 \mathrm{mg}$ daily) was started again, without benefit. A second dermatologist prescribed biotin and topical steroids after a clinical diagnosis of seborrheic dermatitis. After a few days, the patient was hospitalized on emergency due a generalized convulsive episode. MRI contrast imaging documented an isolated, superficial brain lesion, $1 \mathrm{~cm}$ in size, involving the central circumvolution of his right parietal lobe, causing remarkable perilesional edema. A few days later, a contrast CT scan of the brain revealed a hypodense cortical and subcortical lesion at the same site as detected by MRI, with normal appearance of the remaining brain parenchyma. Sampling of CSF documented a mild increase in CSF proteins, whereas PCR and cultural assays for herpes viruses, Toxoplasma gondii and Mycobacterium tuberculosis were negative. His liver function tests worsened, CD4 T-cell counts being $20 / \mathrm{mm}^{3}$. A skin scraping was performed, showing the presence of numerous hyaline septate hyphae. Cultural assays, both on Sabouraud dextrose agar and cycloheximide-added Sabouraud dextrose agar, yielded fungal isolates after a few days of incubation. Macro- and micromorphologic examinations identified the geophilic species $M$. gypseum. Intravenous cotrimoxazole, $70 \mathrm{mg} / \mathrm{kg}$ daily, as well as liposomal amphotericin B, $3 \mathrm{mg} / \mathrm{kg}$ daily, were started suspecting neurotoxoplasmosis and/or cerebral mycosis. A few days later, after evaluation of a GRT for HIV resistance mutations, a salvage HAART regimen was initiated, including enfuvirtide, tipranavir/r, zidovudine, tenofovir and lamivudine. At the ninth week of therapy, when $3 \mathrm{mg} / \mathrm{kg}$ liposomal amphotericin B was still administered 3 times weekly for maintenance, lower limb edemas ensued. Renal function tests revealed serum creatinine at 6.4 $\mathrm{mg} / \mathrm{dl}$, total serum nitrogen at $88 \mathrm{mg} / \mathrm{dl}$, and severe proteinuria $(1,600 \mathrm{mg} / \mathrm{l})$. CD4 T-cell counts were $122 / \mathrm{mm}^{3}$, HIV viremia was undetectable $(<50$ copies $/ \mathrm{ml})$. At this time, the cutaneous lesions had cleared, and his cerebral lesion was still detectable on a control CT scan, although much reduced in size. Tenofovir and liposomal amphotericin B were interrupted, whereas the remaining HAART regimen was continued. Itraconazole was restarted at a dose of $200 \mathrm{mg}$ twice daily. Enfuvirtide was discontinued after 6 additional months. Renal function normalized 40 days after modification of the antiretroviral regimen, and a renal sequential scintigram showed a normal perfusion pattern at the same time; full reversion of proteinuria and peripheral edemas ensued. As of January 2011, approximately 4 years after the acute renal toxicity episode, CD4 T-cell counts were $389 / \mathrm{mm}^{3}$ and HIV viremia was persistently suppressed. 


\begin{tabular}{c|l|l|l}
$\begin{array}{c}\text { Case Reports in } \\
\text { Dermatology }\end{array}$ & $\begin{array}{l}\text { Case Rep Dermatol 2011;3:55-59 } \\
\text { DOI: } 10.1159 / 000326055\end{array}$ & $\begin{array}{l}\text { Published online: } \\
\text { March 4, 2011 }\end{array}$ & $\begin{array}{l}\text { @ 2011 S. Karger AG, Basel } \\
\text { ISSN 1662-6567 } \\
\text { www.karger.com/cde }\end{array}$ \\
\hline
\end{tabular}

\section{Discussion}

We report a case of tinea incognito involving the face and neck in a patient working as a floor-cleaning assistant, with long-lasting HIV infection and severe HIV-related immune depression. First-line systemic treatment with itraconazole was temporarily successful. At relapse of the cutaneous lesions, however, an unsuccessful second-line regimen with topical steroids was administered, based on the eczematous-like appearance of the patient's diffuse facial lesions, in absence of a microbiological characterization. Finally, M. gypseum was isolated from culture of progressed lesions. To our knowledge, this geophilic dermatophyte has been reported in only a few cases of HIV patients so far, and always in resource-poor settings [5-8]. Our case, therefore, is the first case of diffuse tinea incognito due to M. gypseum reported in Western countries in a patient with prolonged professional exposure to soil and dust and severe immune suppression [9].

In our case, tinea incognito, commonly originating from topical corticosteroid treatment, was likely related to the severe and long-lasting depletion and consequent loss of function of CD4 T-lymphocytes. It thus adds to the evidence that dermatological diagnosis should never be based upon the sole appearance of lesions in HIV patients with advanced disease, the contribution of laboratory examination being essential.

Misdiagnosis often leads to an inappropriate use of drugs as topical or systemic corticosteroids, possibly causing further spread of tinea incognito $[4,10]$.

In our patient, skin involvement due to M. gypseum was diagnosed together with a lesion of the right parietal cortex brain causing seizures. Initially, we suspected a CNS infection caused by T. gondii. Brain CT scan findings, however, were atypical for neurotoxoplasmosis, showing no involvement of the basal ganglia and normal ventricular appearance. In addition, the search for T. gondii DNA in CSF by PCR was negative. A broad-spectrum antimicrobial regimen based on cotrimoxazole and liposomal amphotericin B, together with a rescue HAART regimen, led to a reversal of the patient's severe immune suppression and to the clearance of both skin and brain lesions.

Consequently, we cannot exclude that the observed isolated brain lesion might have been caused by M. gypseum itself, being responsive to systemic liposomal amphotericin B and immune rescue.

Our patient had never shown adequate adherence to antiretroviral treatment since he was diagnosed with HIV infection. Interestingly, the occurrence of a state of severe clinical impairment, characterized by combined skin and brain lesions, led to a remarkable and long-lasting increase in adherence to therapy, even during coadministration of a complex antibiotic and antifungal regimen, as well as when severe renal toxicity might have easily discouraged full adherence $[11,12]$.

In conclusion, our case report may represent the first evidence of tinea incognito in a patient with advanced HIV infection and a concomitant reversible CNS lesion, possibly related to the relapse of his cutaneous lesion. Microbiological characterization of skin lesions in advanced HIV patients may play a clinically relevant adjuvant role. 


\begin{tabular}{c|l|l|l}
$\begin{array}{c}\text { Case Reports in } \\
\text { Demmatology }\end{array}$ & $\begin{array}{l}\text { Case Rep Dermatol 2011;3:55-59 } \\
\text { DOI: } 10.1159 / 000326055\end{array}$ & $\begin{array}{l}\text { Published online: } \\
\text { March 4, 2011 }\end{array}$ & $\begin{array}{l}\text { O 2011 S. Karger AG, Basel } \\
\text { ISSN 1662-6567 } \\
\text { www.karger.com/cde }\end{array}$ \\
\hline
\end{tabular}

\section{Acknowledgements}

We are indebted to Mr. Vincenzo Massei, Mrs. Loredana Puglielli and Angela Pisciella for their invaluable contribution in the clinical management of the patient.

\section{Disclosure Statement}

None of the authors has any conflict of interest to declare. E.P. was funded by a grant from the Fondazione Camillo de Lellis per l'Innovazione e la Ricerca in Medicina, Pescara, Italy.

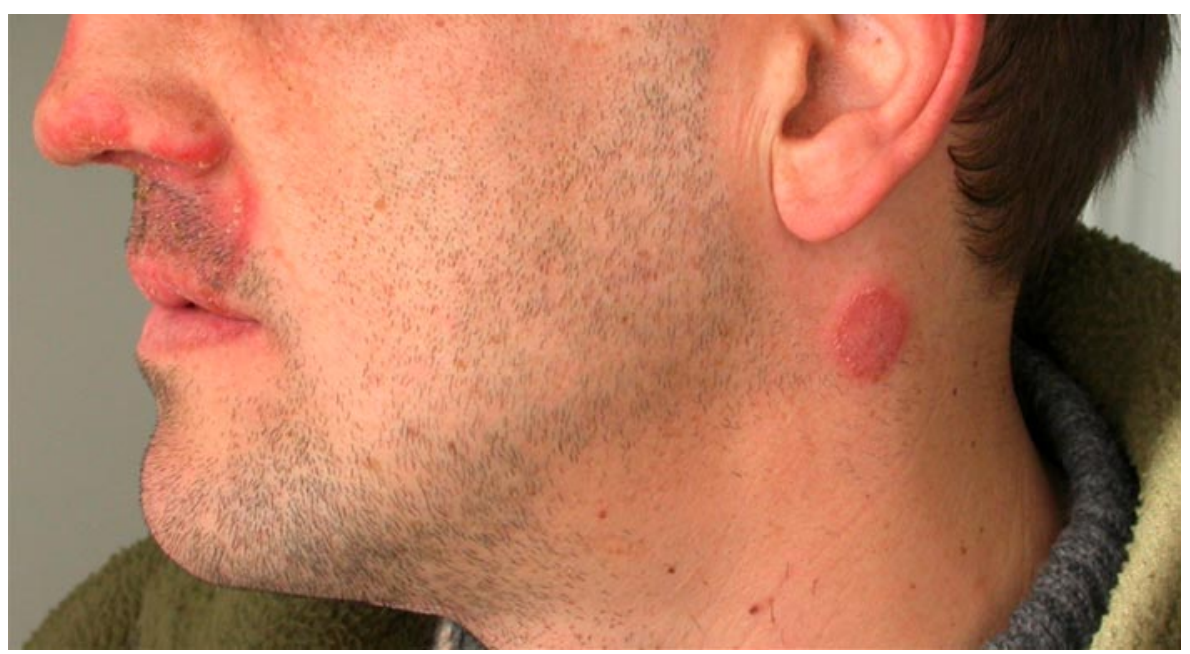

Fig. 1. Picture of the face and neck of the patient taken at the time of the first diagnosis of dermatophytosis. Lesions appeared as two ring-shaped, erythematous scaly plaques with inflammatory advancing borders located on the upper lip, nose and side of the neck. 


\begin{tabular}{c|l|l|l}
$\begin{array}{c}\text { Case Reports in } \\
\text { Dermatology }\end{array}$ & $\begin{array}{l}\text { Case Rep Dermatol 2011;3:55-59 } \\
\text { DOI: } 10.1159 / 000326055\end{array}$ & $\begin{array}{l}\text { Published online: } \\
\text { March 4, 2011 }\end{array}$ & $\begin{array}{l}\text { O 2011 S. Karger AG, Basel } \\
\text { ISSN 1662-6567 } \\
\text { www.karger.com/cde }\end{array}$ \\
\hline
\end{tabular}
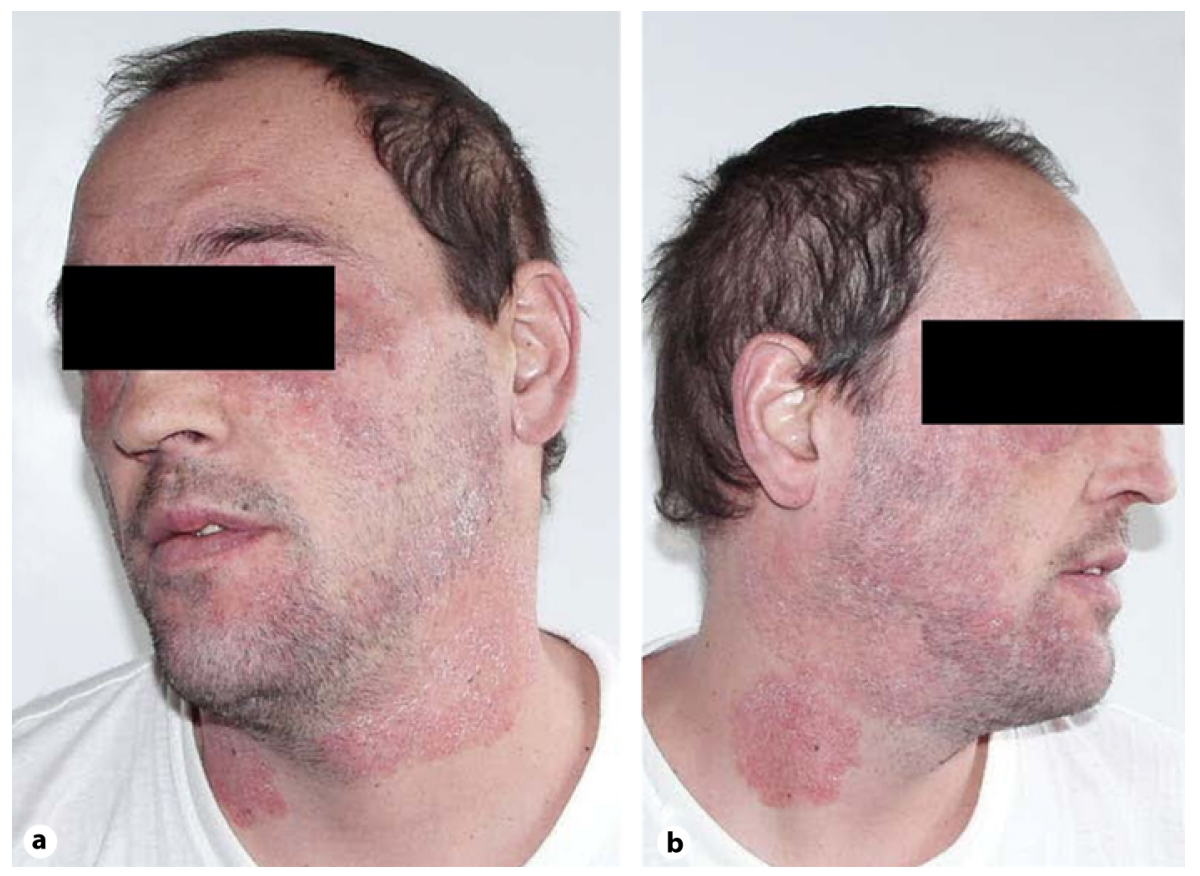

Fig. 2. Lesions on the neck and face of the patient at the time of relapse, approximately six months after the first diagnosis. They consist of ill-defined, confluent, scantly inflammatory erythematous scaly patches, diffusely involving the face and neck.

\section{References}

1 Gupta AK, Cooper EA, Ryder JE, Nicol KA, Chow M, Chaudhry MM: Optimal management of fungal infections of the skin, hair, and nails. Am J Clin Dermatol 2004;5:225-237.

$\checkmark 2$ Johnson RA: HIV disease: mucocutaneous fungal infections in HIV disease. Clin Dermatol 2000;18:411-422.

3 Romano C, Maritati E, Gianni C: Tinea incognito in Italy: a 15-year survey. Mycoses 2006;49:383-387.

-4 Arenas R, Moreno-Coutiño G, Vera L, Welsh O: Tinea incognito. Clin Dermatol 2010;28:137-139.

5 Galhardo MC, Wanke B, Reis RS, Oliveira LA, Valle AC: Disseminated dermatophytosis caused by Microsporum gypseum in an AIDS patient: response to terbinafien and amorolfine. Mycoses 2004;47:238-241.

6 Luque AG, Biasoli MS, Sortino MA, Lupo SH, Bussy RF: Atypical tinea corporis caused by Microsporum gypseum in a subject with acquired immune deficiency syndrome. J Eur Acad Dermatol Venereol 2001;15:374375.

7 Porro AM, Yoshioka MC, Kaminski SK, Palmeira Mdo C, Fischman O, Alchorne MM: Disseminated dermatophytosis caused by Microsporum gypseum in two patients with the acquired immunodeficiency syndrome. Mycopathologia 1997;137:9-12.

8 Prochnau A, de Almeida HL Jr, Souza PR, Vetoratto G, Duquia RP, Defferrari R: Scutular tinea of the scrotum: report of two cases. Mycoses 2005;48:162-164.

-9 Romano C, Massai L, Gallo A, Fimiani M: Microsporum gypseum infection in the Siena area in 2005-2006. Mycoses 2009;52:67-71.

10 Ive FA, Marks R: Tinea incognito. Br Med J 1968;3:149-152.

11 Ter Heine R, Huitema AD, Jansen RS, Smits PH, van Gorp EC, Wagenaar JF, Beijnen JH, Mulder JW: Prolonged exposure to tenofovir monotherapy 1 month after treatment discontinuation because of tenofovirrelated renal failure. Antivir Ther 2009;14:299-301.

12 Lanternier F, Lortholary O: Liposomal amphotericin B: what is its role in 2008? Clin Microbiol Infect 2008;14(suppl 4):71-83. 